\title{
Circular background decreases misunderstanding of multidimensional scaling results for naive readers
}

\author{
$F$. Degret, and $S$. Lespinats* \\ Univ. Grenoble Alpes, INES, F-73375, Le Bourget du Lac, France.
}

\begin{abstract}
Non-linear multidimensional scaling (NL-MDS) methods are widely used to give an insight on structures of a dataset. Such a technic displays a "map" of data points onto a 2 dimensional space. The reader is expected to have natural understanding of proximity relationships between items. In our experience, MDS are especially helpful as a support for the collaboration between data analysts and specialists of other fields. Indeed, it often allows understanding main issues, major features, how to deal with data and so on. However, we observed that the classical/rectangular display of map causes confusion for non-specialists and long explanation is often required before reaching the fruitful step of the collaboration. The meaning -the absence of meaning, actually- of axes can be subject for many questions and skepticism from many naive persons. Although it is hardly quantifiable, we observed that using a circle-shaped background for maps improves the understanding of the concept of data mapping by far. We however present here a subjective feedback that may support the practical contribution of NL-MDS for other scientific fields.
\end{abstract}

\section{NL-MDS aims at providing information about data organisation}

Multi-dimensional scaling (MDS) methods can be defined as the set of methods that display data lying in a metric space (taken into account through a distance matrix) onto an output space while preserving "as much as possible" the distances between them. Of course, there are many ways to set the meaning of "as much as possible". For example, the classical MDS [1] minimizes the raw square difference between original and output distances when non-linear methods (NL-MDS) assign a higher weight to short distances in order to favor there preservation, which, consequently relax constraints on larger distances. There are a huge number of existing methods proposed in that goal, including Sammon's mapping [2], Curvilinear Component Analysis [3], Isomap [4], Locally Linear Embedding [5], Local MultiDimensional Scaling [6], Data-Driven High Dimensional Scaling [7] and Stochastic Neighbor Embedding [8, 9] and NeRV [10].

Most often, the output space is a two (or three) dimensional Euclidean space in order to allow an intuitive exploration. Indeed, most of the time, such algorithms aim at providing a better understanding of the dataset. In the present paper, we focus on cases where the output

\footnotetext{
*Corresponding author: sylvain.lespinats@cea.fr
} 
space is bidimensional. The proposed solution is unfortunately hardly extendable to tridimensional maps.

The present paper in organized as follow: the current section 1 introduces multidimensional scaling concept. The second section explains why non-linear multidimensional scalings are often hard to read for users that are non-familiar with such methods. Section 3 propose a simple and intuitive solution that seems to help the users to infer from non-linear maps. Section 4 is dedicated to conclusions and perspectives.

\section{Misunderstanding of a display originally made to be intuitive}

In the following, "naïve reader" refer to a person that:

- does not have been exposed to data maps reached with multidimensional scaling;

- does not currently understand the concept of "data map" and need explanation to infer from it;

- and (obviously) does not know how it is calculated.

As stated in section 1, the main purpose of NL-MDS is the display of a dataset so as to give an intuitive insight on the data structure, even for a naive reader. Complex configurations in a high dimensional space is expected to become easily observable. The algorithm's result can be seen as a "map" of the data and, by extension, NL-MDS methods are often called "mapping methods". As a geographical map, its orientation is no more than a convention (just as placing north at the top of a geographical map). In data maps, the x- and y-axes have not particular meaning: every rotation or symmetry is also validate. Indeed, the only relevant information lies in the distances displayed between items, that is to say where items are, according to other ones.

However, although the absence of axes meaning is somewhat obvious for data analysts who know how maps are calculated, we learned from experience that it seems to be very uncomfortable for other ones. Most of the time, peoples face uncommon/hard to understand figures. The figure 1 - insert A - shows a typical example. In such a case, the most spontaneous intellectual process to overcome the obstacle is figuring out the meaning of axes. In our experience, this question is almost systematical asked by naive readers when a map in shown, whatever the previous speaker's explanations. Obviously, there is a shamed paradox in the fact that so laborious explanations are needed for graphics originally designed to be intuitive.

\section{Hiding axes helps to focus on relevant information}

Because axes are confusing and does not bring information they are sometime discarded from figures drawn by NL-MDS and replaced by a bar scale (as in a geographical map, see figure 1 - insert $\mathrm{B}-$ ). We observed that doing so does not help much because the rectangular frame of the figure is still understood as axes by the reader. Discarding the frame does not help neither: the shape of the support (screen, slide, sheet of paper, etc) plays the frame role in the reader's eye. However, we noticed that switching the rectangular frame by a circular one (figure 1 - insert $\mathrm{C}-$ ) changes the reaction: axes totally disappear and the attention of the reader seems to be drove toward relevant information. 

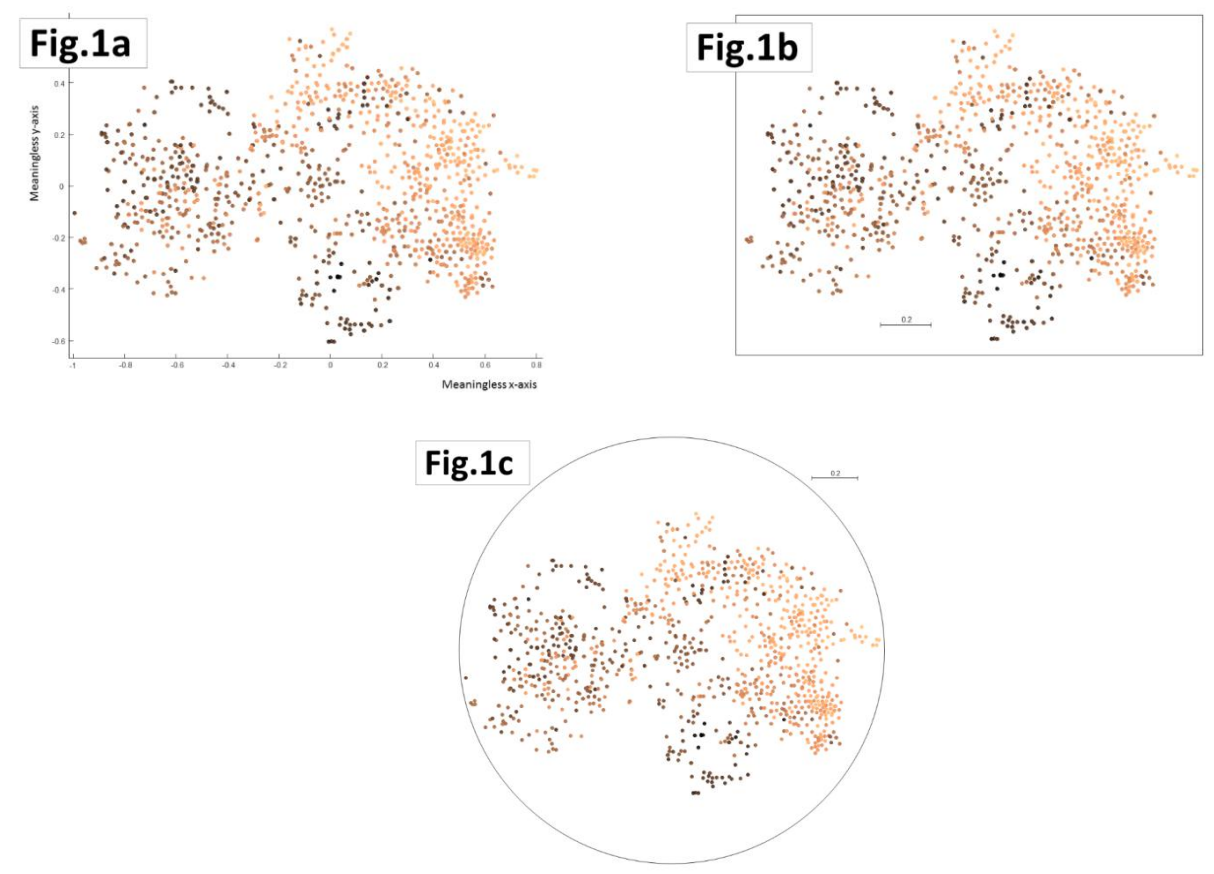

Fig. 1. An example of map used as a basis for discussion. Each item codes for an acoustic emission in a $\mathrm{Ni}-\mathrm{Zn}$ battery. Color displays the average frequency which is one of the significant features describing an acoustic burst (dark for high frequencies). This map has been performed so as to increase our understanding of the various types of signal (resulting from various physico-chemical phenomena). In inserts $\mathrm{A}, \mathrm{B}$ and $\mathrm{C}$, the map is exactly (from position and color points of view) the same but the background and scale display is modified.

Fig. 1a. Classical scatter plot with scaled $x$ - and y-axes. Naive readers often wonder the meaning of axes although it is meaningless.

Fig. 1b. Because axes haves no particular meaning, the map is sometimes presented while hiding axes. A bar scale presents the ratio between a distance in the original space and onto the map. Naive readers often still take the rectangular frame for axes.

Fig. 1c. A circular frame seems to solve such an issue. The bar scale is also displayed. The owning of the map by naive readers seems to be easier.

In our experience, there are much less questions on how to infer from the map with circular background, and the time needed for being familiar with map concept decreases by far. We should however notice that these elements have not been quantified and must be taken as nothing more than our personal feedback.

Our solution may be related to 3 previous works:

- the Onclinx's mapping [11], where data are mapped onto the surface of a 3 dimensional sphere rather than a plan;

- the H-MDS [12], where the output space is hyperbolic (which is drawn circular) and

- Colorphylo [13], where a data map is related to a circular slice of the HSV colorspace in order to provide a color-code associated to the map.

The circular background positioning may be done "by hand". The smallest circle that contains all the data can also be calculated (it should be noticed that the Jung's theorem [14] gives a maximum bound for its radius). The following 2-steps algorithm may be used in that goal:

1) The first step determines the smallest convex polygon that contains every data. The first point of the polygon is one of the ends of the maximum distances in the output space. Then, a straight line between the last-chosen-point for the polygon and every 
ones of not-already-chosen-points is drawn. The point associated to the line that does separate the dataset in two parts (that is to say, every points lie in the same side of the line) is chosen to complete the polygon. This stage is repeated until that every points lie inside the closed polygon. The first step's complexity is $\mathrm{O}(\mathrm{N})$ where $\mathrm{N}$ is the number of items in the dataset.

2) The second step determines the smallest encompassing circle (i.e. the smallest circle that contains the dataset). As this encompassing circle is also the smallest circle that contains the summits of the polygon determined in step 1, all other items are ignored in this step. Each combination of 3 summits is now considered. The associated circumcircle of the corresponding triangle can be easily calculated (the circumcenter is positioned at the crossing of the three perpendicular bisectors of the triangle). If every other summits fall inside the circumcircle (i.e. every distances to the center are inferior or equal to the circle radius), the circumcircle is candidate for being the smallest encompassing circle. The candidate circle that have the smallest radius is chosen. The step 2 complexity is $\mathrm{O}\left(\mathrm{N}_{1}{ }^{3}\right)$ where $\mathrm{N}_{1}$ is the number of summits of the polygon (which is supposed to be small with respect to $\mathrm{N}$ ).

\section{Conclusions and perspectives}

The present paper describes issues often met in real life when presenting data maps reached by NL-MDS to naive readers. It introduces an empirical solution: changing the background's shape so as to sweep the concept of axis away from the reader's mind.

A quantitative evaluation of the solution is obviously desirable and is planned. Such an evaluation may be done by presenting a map to a set of naive readers. The readability of maps according to the background's shape could be evaluated by the number of asked questions and/or the time spend before inferring. However, it is clearly uneasy to do and remains to set up. Several issues are to be solved:

What is the meaning of being naive? How to constitute the readers pool? Does a tested reader should have scientific skills or should we perform tests on anybody (which will maybe not have the same "reflex" of seeking for axes)?

How to account for the experimenter effect? Indeed, the experimenter have to explain the map and how to read it. Obviously, the teaching skill of the experimenter will affect the results.

Moreover, the both cases cannot be tested on the same reader. A reader must not be considered as "naive" more than one single time. The understanding of one single reader on the both shapes of background cannot be compared. This negatively affect the statistical power of the test and must be counterbalance by a large number of experiments.

We rather believe that questioning the data analysts which will use circular background as suggested here could be a better test. Indeed their subjective feeling on the influence of background on the readers' understanding may be quantified on a scale and be statistically evaluated thereafter.

However, it is worth to notice that we could observe every one of the 4 battery experts with which we collaborate reported that they were at first strongly confused with usual rectangular maps. They approved the circular shape and reported that it is clearly more intuitive.

\section{Acknowledgments}

The author would like to thank the Bpifrance and the "Commissariat à l'énergie atomique et aux énergies alternatives" for his financial support. 


\section{References}

1. Torgerson W.S., 1952. Multidimensional scaling: 1. Theory and method. Psychometrika, 17: 401-419.

2. Sammon, J.W., 1969. A nonlinear mapping for data structure analysis, IEEE Trans. Comput., vol. C-18, no. 5, pp. 401-409.

3. Demartines, P., Hérault J., 1997. Curvilinear component analysis: A selforganizing neural network for nonlinear mapping of data sets, IEEE Trans. Neural Netw., vol. 8, no. 1, pp. 148-154.

4. Tenenbaum, J.B., De Silva, V., Langford, J.C., 2000. A global geometric framework for nonlinear dimensionality reduction. Science, 290: 2319-2323.

5. Roweis, S. T., \& Saul, L. K., 2000. Nonlinear dimensionality reduction by locally linear embedding. Science, 290(5500), 2323-2326.

6. Venna J., Kaski S., 2006. Local multidimensional scaling, Neural Networks, vol. 19, no. 6-7, pp. 889-899.

7. Lespinats, S., Verleysen, M., Giron, A., Fertil, B., 2007. DD-HDS: a tool for visualization and exploration of highdimensional data, IEEE Trans. Neural Netw., vol. 18, no. 5, pp. 1265-1279.

8. Hinton, G., Roweis, S., 2002. Stochastic neighbor embedding, in: NIPS, vol. 15, pp. 833-840.

9. Van der Maaten, L., \& Hinton, G., 2008. Visualizing data using t-SNE. Journal of Machine Learning Research, 9(2579-2605), 85.

10. Venna, J., Peltonen, J., Nybo, K., Aidos, H., \& Kaski, S. (2010). Information retrieval perspective to nonlinear dimensionality reduction for data visualization. Journal of Machine Learning Research, 11(Feb), 451-490.

11. Onclinx, V., Lee, J. A., Wertz, V., Verleysen, M., 2010. Dimensionality reduction by rank preservation. In Neural Networks (IJCNN), The 2010 International Joint Conference on (pp. 1-8). IEEE.

12. Walter, J.A., 2004. H-MDS: a new approach for interactive visualization with multidimensional scaling in the hyperbolic space. Information systems, 29(4), 273-292.

13. Lespinats, S., Fertil, B. (2011) "A color code to accurately account for taxonomic classification." Evolutionary Bioinformatics 2011:7 257-270.

14. Jung, H., 1901. "Über die kleinste Kugel, die eine räumliche Figur einschließt". J. Reine Angew. Math. 123: 241-257. 